\title{
Evaluation einer ähnlichkeitsbasierten Produktsuche für Kleidung
}

\author{
Alexander Piazza, Christian Zagel, Sebastian Huber, Jana Bernotat \\ Lehrstuhl für Wirtschaftsinformatik, insbes. im Dienstleistungsbereich, Friedrich-Alexander- \\ Universität Erlangen-Nürnberg
}

\section{Zusammenfassung}

Das Produktangebot in Handels- und Onlineumgebungen überfordert den Kunden zunehmend. Aktuelle Untersuchungen zeigen, dass sich zu viele Auswahlmöglichkeiten negativ auf die Einkaufserfahrung auswirken können. Um dem entgegenzuwirken werden z. B. in der Modeindustrie Self-ServiceTerminals eingesetzt, die es dem Kunden erlauben, das Produktangebot zu filtern. Diese Ansätze funktionieren jedoch nur bei Kenntnis der eigenen Präferenzen. Abhilfe schaffen hier sog. ähnlichkeitsbasierte Produktsuchen. Dieser Beitrag stellt die Ergebnisse eines Pretests zur Nutzerakzeptanz für ein innovatives Bedienkonzept vor, welches es erlaubt intuitiv durch Produktangebote auf Basis visueller Präferenzen zu navigieren. Der Lösungsvorschlag basiert auf visuellen Unterschieden, die aus Produktbildern extrahiert werden können. Die Eignung dieses neuartigen Ansatzes wird abschließend in Form einer Benutzerstudie evaluiert.

\section{Einleitung}

Motiviert wird diese Studie durch die Beobachtung, dass sich Benutzeroberflächen in Online-Läden für Kleidungsartikel auf klassische Filter- und Sortierfunktionalitäten beschränken, mit denen Kunden die angebotene Produktpalette durchsuchen können. Die Annahme in dieser Forschung ist hierbei, dass diese Funktionalitäten geeignet sind für Kunden, die schon eine konkrete Vorstellung für deren Präferenzen besitzen. Falls sich die Kunden jedoch noch in der Phase der Präferenzbildung befinden, bei der sie sich anhand der angebotenen Produkte inspirieren lassen, können noch keine konkreten Filter angegeben werden. Das Ziel dieser Untersuchung ist es zu evaluieren in welchem Grad ähnlichkeitsbasierte Produktsuchen diesen Prozess unterstützen können. Bei dieser Produktsuche geben die Benutzer nicht primär Präferenzen in Form von Filter- und Sortierregeln an, sondern indizieren die Präferenz durch das Klicken auf ein Produkt. Anschließend werden Produkte angezeigt, die ein ähnliches Aussehen besitzen. 
Um diesen Ansatz zu untersuchen, wurde ein Prototyp der Produktvisualisierung basierend auf Webtechnologien entwickelt. In (Piazza et al. in Print) wurde ein Pretest vorgestellt der zur Akzeptanz der Lösung im Rahmen einer simulierten Im-Laden Situation im Labor durchgeführt wurde. Hierbei wurde der Prototyp auf einem lebensgroßen Touch-Display projiziert und 36 Probanden eingeladen die Visualisierung zu nutzen. Dieser Pretest ergab, dass die Nutzer generell positiv auf diese Art von Nutzeroberfläche im Laden reagierten. Ziel der vorliegenden Studie ist es, im Rahmen eines zweiten Pretests die Akzeptanz dieser Visualisierung im Kontext des Online-Handels zu evaluieren.

\section{Aufbau der Visualisierung}

Die grundlegende Idee dieser Visualisierung ist es eine Navigation anzubieten, die ausschließlich auf Produktähnlichkeiten beruht und außer der Auswahl von Geschlecht und Primärfarbe des Kleidungsstücks keine Filtermöglichkeit anbietet.

Hierfür müssen zunächst die Attribute zum Aussehen der Kleidungsprodukte aus den Produktbildern extrahiert werden. Grundlage für diese Studie bilden ca. 3000 Produktbilder eines deutschen Online-Händlers. Bei der Extraktion der Attribute wird zunächst der Hintergrund des Produktbildes segmentiert und anschließend die Primärfarbe bestimmt. Für diese Farbe wird anschließend die Farbtemperatur, Helligkeit und der Kontrast ermittelt. Das Vorgehen zur Ermittlung dieser Parameter ist in (Piazza et al. in Print) beschrieben.

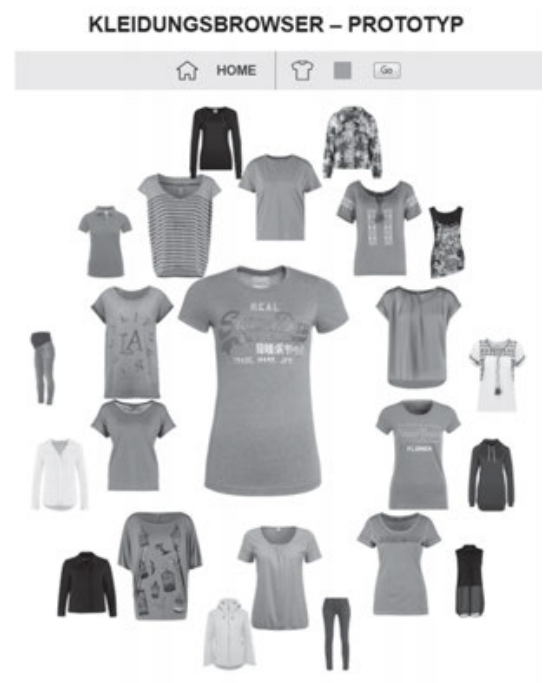

Figure 1: Aufbau der Produktvisualisierung

Auf Basis der Produktfarbe wird anschließend für jedes Produkt eine Auswahl an ähnlichsten Artikel ermittelt und visualisiert. Die Benutzerinteraktion gestaltet sich wie folgt: In der Bildschirmmitte ist das derzeit ausgewählte Produkt zu sehen. Unmittelbar um das ausge- 
wählte Produkt werden die Empfehlungen mit der ähnlichsten Farbe angezeigt. Nach außen hin nimmt der Grad der Ähnlichkeit stetig ab, um den Benutzer auch neue Inspirationen geben zu können. Bei Auswahl eines Produkts berechnet der Empfehlungsalgorithmus neue Vorschläge und passt die Visualisierung entsprechend an. Mit diesem Ansatz kann der Kunde schnell das Produktsortiment durchsuchen, ohne sich dabei durch lange Produktlisten klicken zu müssen, sowie ohne die Angabe von expliziten Filtern.

\section{Evaluation}

Im Rahmen einer Online-Umfrage wurden Teilnehmer eingeladen den Prototypen zu nutzen und sowohl quantitativ als auch qualitativ eine Einschätzung zur Eignung des Prototyps zu geben. Quantitativ wird hierbei einerseits die wahrgenommene Nützlichkeit orientiert an dem Vorgehen von (Stüber 2013) erhoben, sowie die Service Faszination nach (Zagel \& Bodendorf 2014). Die Fragen wurden mit einer siebenstufigen Likert-Skala bewertet, mit den Endpunkten 1 = ,trifft überhaupt nicht zu“ und 7 = ,trifft voll und ganz zu“. Zudem wurde qualitativ gefragt, welche generelle Meinung die Teilnehmer zur Visualisierung haben und welches Verbesserungspotenzial gesehen wird. Insgesamt haben 82 Teilnehmer in einem Zeitfenster von ca. zwei Wochen an der Umfrage teilgenommen. Dabei sind 57 Teilnehmer weiblich und 25 Männlich. Das Durchschnittsalter ist 26,42 mit einer Standardabweichung von 9,70 .

\begin{tabular}{|c|c|c|c|c|}
\hline Service Faszination & $\mathrm{Md}$ & $\mathrm{X}$ & SD & Basierend auf \\
\hline $\begin{array}{l}\text { Ich würde anderen von meiner positiven Erfahrung der } \\
\text { Nutzung des Systems erzählen. }\end{array}$ & 4 & 4,0 & 1,94 & (Maxham 2001) \\
\hline Ich würde die Nutzung des Systems empfehlen. & 4 & 3,95 & 1,85 & (Maxham 2001) \\
\hline Die Nutzung des Systems ist aufregend. & 4 & 3,65 & 1,57 & (Childers et al 2001) \\
\hline $\begin{array}{l}\text { Ich bin mir sicher, dass ich das System nutzen würde, wenn } \\
\text { ich die Möglichkeit hätte. }\end{array}$ & 4 & 4,91 & 1,77 & $\begin{array}{l}\text { (Venkatesh \& Davis } \\
\text { 2000) }\end{array}$ \\
\hline Ich werde das System in Zukunft wiederholt nutzen. & 3 & 3,51 & 1,81 & (Gu et all 2010) \\
\hline Wahrgenommene Nützlichkeit & $\mathrm{Md}$ & $\mathrm{x}$ & SD & Basierend auf \\
\hline \multicolumn{5}{|l|}{ Durch die Anwendung der Kleidungsvisualisierung... } \\
\hline ...ist eine schnellere Auswahl möglich & 4 & 4,29 & 1,65 & (Stüber 2013) \\
\hline ...wird der Suchaufwand verringert. & 4 & 4,12 & 1,76 & (Stüber 2013) \\
\hline ...kann das Einkaufsergebnis verbessert werden. & 5 & 4,29 & 1,78 & (Stüber 2013) \\
\hline
\end{tabular}

Tabelle 1: Ergebnisse der quantitativen Befragung

Die quantitativen Ergebnisse der Umfrage sind in Tabelle 1 aufgeführt und zeigen eine moderat positive Akzeptanz der neuartigen Produktvisualisierung. Insgesamt haben 69 Teilnehmer qualitatives Feedback zur Umfrage gegeben. Hierbei wird vor allem genannt, dass sich die Benutzer sich mehr Sortier- und Filtermöglichkeiten wünschen. Außerdem wird die Genauigkeit der Ähnlichkeitssuche des Empfehlungsalgorithmus bemängeln. 


\section{Zusammenfassung \& Ausblick}

Das Ziel dieser Studie war es, die Nutzerakzeptanz eines Prototyps für die Visualisierung von Kleidung basierend auf Produktähnlichkeiten im Kontext einer Onlinenutzung zu evaluieren. Die Ergebnisse der Studie deuten darauf hin, dass die Teilnehmer der Untersuchung einem derartigen Konzept generell positiv gegenüberstehen. Entgegen der ursprünglichen Vermutung scheint eine primär auf Produktähnlichkeit basierende Oberfläche bei den Nutzern als nicht ausreichend angesehen zu werden. Es werden zusätzlich Filter- und Sortierfunktionalitäten erwartet. Aufbauend auf diesen Zwischenergebnissen wird die Präzision der Ähnlichkeitssuche durch den Einsatz von zusätzlichen Bildvergleichsverfahren erweitert, wie beispielsweise der Vergleich basierend auf Farbhistogrammen statt der Primärfarbe. Zudem werden weitere Funktionalitäten zum Filtern und Sortieren der Produkte implementiert und anschließend die Akzeptanz anhand einer Nutzerstudie evaluiert.

\section{Danksagung}

Besonderer Dank gilt der „Hans-Frisch-Stiftung“ für die Förderung des Projekts.

\section{Literaturverzeichnis}

Childers L. T., Carr, L. C., Peck J., \& Carson, S. (2001). Hedonic and utilitarian motivations for online retail shopping behavior. Journal of Retailing 77, 511-535.

Gu, J.-C., Fan, L., Suh, Y. H., \& Lee, S.-C. (2010). Comparing Utilitarian and Hedonic Usefulness to User Intention in Multipurpose Information Systems. Cyberpsychology, Behavior, and Social Networking 13, 287-297.

Maxham, J. G. (2001). Service recovery's influence on consumer satisfaction, positive word-of-mouth, and purchase intentions. Journal of Business Research 54, 11-24.

Piazza, A., Zagel, C., Huber S., \& Hille, M. (in print). Outfit Browser: An Image-Data-Driven User Interface for Self-Service Systems in Fashion Stores. In: Advances in The Human Side of Service Engineering. AHFE Conference 2015, Las Vegas, USA, p. in print.

Stüber, E. (2013). Personalisierung im Internethandel - Die Akzeptanz von Kaufempfehlungen in der Bekleidungsbranche. Wiesbaden: Gabler Verlag.

Venkatesh, V., \& Davis, F. D. (2000). A Theoretical Extension of the Technology Acceptance Model: Four Longitudinal Field Studies. Management Science 46, 186-204.

Zagel, C., \& Bodendorf, F. (2014). Success factors and approaches of service fascination - A research framework, in: Freund, L., \& Cellary, W. (Eds.), Advances in the Human Side of Service Engineering, AHFE Conference, 2014, 537-548.

\section{Kontaktinformationen}

Alexander Piazza, Christian Zagel, Sebastian Huber

Lehrstuhl für Wirtschaftsinformatik, insbes. im Dienstleistungsbereich, Friedrich-Alexander Universität Erlangen-Nürnberg, Lange Gasse 20, 90403 Nürnberg

alexander.piazza@fau.de 